Hans-Jürgen P. Walter

\title{
Nicolai Hartmanns Kritische Ontologie („wie sie als Grundlage der Gnoseologie anzustreben ist ") und der Kritische Realismus der Gestaltpsychologie („Berliner Schule“/Gestalttheorie)
}

\section{Ein erfundener Dialog über eine ungeschriebene Dissertation}

\section{Teil ${ }^{1}$}

\section{Vorbemerkungen}

Vor fünf Jahren erfand ich meinen Enkelsohn Adrian und begann mit ihm meine „Gespräche mit Adrian über Gott und die Welt“. Beim ersten Gespräch war er 13 Jahre alt. Meine real existierenden Enkelkinder waren zu diesem Zeitpunkt erst zwischen 3 und 10 Jahren alt. Ich gestehe, dass Versuche vorangegangen waren, mit den beiden ältesten Enkelsöhnen - der jüngere von ihnen war 7 Jahre alt in fortlaufende Gespräche einzutreten, die beiderseitigem Interesse entsprachen, nicht von Erfolg gekrönt waren. Vielleicht sahen wir uns auch nur zu selten, um über irgendwelche „kindgerechten“ gemeinsamen Unternehmungen hinauskommen zu können. Vielleicht war meine Intention, mit einem Kind über grundlegende Fragen menschlichen Glaubens, Denkens und Handelns zu sprechen auch einfach zu anspruchsvoll. Vielleicht überschätzte ich in der Erinnerung schon den 8-10-jährigen Pfarrerssohn, der ich einmal war.

Gewissermaßen ersatzweise verfasste ich - ohne die erhoffte Enkel-Hilfe - eine Lehr-Erzählung, in der 3 Neunjährige an das graphisch ansprechend gestaltete Buch „Schöpferisch träumen“ von Paul Tholey und Kaleb Utecht (FalkenVerlag,1987) geraten und sich daran machen, das reich bebilderte 5. Kapitel (S. 134-154) „Erkenntnistheorie für den Hausgebrauch“ zu verstehen. In ihrer 4. Schulklasse halten sie am Ende sehr erfolgreich einen Vortrag über „optische Täuschungen“. Immerhin gönnte mir mein jetzt 12-jähriger Enkel beim jüngsten Besuch die Genugtuung, das Heftchen in zwei Tagen zu Ende zu lesen und die Geschichte „teilweise lustig“ zu finden.

Wie gesagt, erfand ich schließlich meinen 13-jährigen Enkelsohn Adrian. Das erste Gespräch beginnt damit, dass er mich besucht und bei einem Spaziergang

\footnotetext{
${ }^{1}$ Der 2. und letzte Teil des Beitrags wird in der nächsten Ausgabe der Gestalt Theory (1/2019) erscheinen. 
unvermittelt fragt: „Opa, glaubst Du eigentlich an Gott?“ Religiöse Fragen bleiben für mehrere Besuche im Mittelpunkt unserer Gespräche. Ich lasse ihn relativ schnell 10 Jahre älter werden. Mit 23 Jahren hat Adrian ein wirtschaftswissenschaftliches Studium abgeschlossen, ,jobbt“, anscheinend ganz erfolgreich, und studiert neuerdings weiter: Philosophie. Einerseits geht es um weitere Präzisierung in der Vergegenwärtigung der Gestalttheorie, andererseits spitzt sich die religiöse Fragestellung auf die theologische Erörterung von Unterschieden zwischen „Gott-ist-Mitmenschlichkeit-Glaube“ (Herbert Braun) und „Offenbarungs-Glaube“ (Karl Barth, Helmut Gollwitzer) zu. Adrian und Opa verständigen sich darauf:

Mit Offenbarung Gottes ist nicht gemeint, dass der daran Glaubende erfährt, wer Gott ist. Gott bleibt, wie Gollwitzer sagt, ,in einem unvergleichlichen Maße von uns unterschieden..., nämlich als der Schöpfer vom Geschöpf" (Gollwitzer, 1963, S. 105). Wir können ihm nur in dem Ausschnitt begegnen, den er uns schenkt; das ist für Christen zuallererst Jesus. Der Kern des Offenbarungsglaubens ist, im Gegensatz zu landläufigem Verständnis, gerade nicht der Glaube, über Gott und das Jenseits Bescheid zu wissen, sondern das Eingeständnis, dass wir nichts davon wissen, warum es die Welt und uns in dieser Welt überhaupt gibt, und dass wir auch über Gott nichts wissen können. Und wenn es einen Himmel gäbe, so hätten wir auch keine Ahnung, was er ist und wer hineinkäme und wer nicht. Wir haben keine Ahnung, warum der Mensch die Freiheit hat, seinesgleichen erbarmungslos zu quälen. Wir können es nicht verstehen, weil wir weder Anfang noch Ende der Welt kennen.

Das zweieinhalb Jahre später stattfindende Gespräch über Adrians philosophische Dissertation knüpft an die theologische Erörterung an. Von literarisch-belletristischen Ansprüchen an die Dialogform bitte ich abzusehen. Sie hat mir die Arbeit erleichtert und dient, wie ich hoffe, der Allgemeinverständlichkeit.

\section{Das Gespräch mit Adrian über seine Dissertation mit dem Arbeitstitel}

„Erkenntnistheoretische und ontologische Grundlagen bei Nicolai Hartmann und der Gestaltpsychologie der ,Berliner Schule‘ (Gestalttheorie) im Vergleich"

„So, Opa“, eröffnete Adrian das Gespräch, „das hast Du jetzt davon, dass Du mich auf Nicolai Hartmann (NH) neugierig gemacht hast. Jetzt bin ich Atheist geworden!“

„Ach was“, sagte ich.

„Was heißt ,Ach was'?“ 
„Dass ich das nicht glaube, heißt das. Jemand, der halbwegs begriffen hat, was „Offenbarungstheologie“ im Sinne Karl Barths und Helmut Gollwitzers bedeutet, mag die Schlüssigkeit welcher religiösen Erscheinungsformen auch immer anzweifeln, er wird deshalb doch kein „Atheist“. Das wäre dumm, und dumm bist Du nicht! Du weißt zu gut, dass Du über den, geglaubten Gott' nichts, jedenfalls nichts Endgültiges, weißt; und Wissen über den ,Gott des Glaubens' ist nichts anderes als das Wissen davon, was Hinz und Kunz so über Gott denken. Ganz abgesehen davon, dass die Behauptung, es könne keinen Gott geben, auch von der Gestalttheorie (Berliner Schule der Gestaltpsychologie) nicht vertreten wird."

„Da will ich Dich mal foppen; und dann fährst Du gleich ganz schwere Kaliber auf. Also, ich bin aber ,methodischer Atheist' ."

„Nachtigall, ick hör Dir trapsen'. Du hast den Vorbesitzer der Hartmann-Bände, die ich uns in einem Marburger Antiquariat - übrigens zu einem bemerkenswert günstigen Preis - besorgt habe, gegoogelt und bei Wikipedia erfahren, dass sich der Religionswissenschaftler Prof. Dr. mult. Dr. h.c. mult. Kurt Rudolph als ,methodischer Atheist' bezeichnet."

„So war's. Aber Du müsstest doch zumindest gesehen haben, dass Stegmüller (1975) NH als Atheisten einsortiert."

„Diese Behauptung hab ich auch noch bei einem anderen philosophischen Schwätzer gelesen. Anscheinend ist unter oberflächlichen Philosophen das Vorurteil verbreitet, dass, wer den ,Idealismus' in der Philosophie (soweit er die Existenz eines Schöpfergottes oder Weltgeistes voraussetzt) ablehnt, ,Atheist` sein müsse. $\mathrm{NH}$ ist allerdings ein großer Entmythologisierer, könnte man sagen, der Philosophie (s. u.a. sein Buch ,Teleologisches Denken' (1966)), durchaus vergleichbar mit dem berühmten theologischen Entmythologisierer Rudolf Bultmann, dem er in seiner Marburger Zeit freundschaftlich verbunden war. Nun hast Du ja NHs dicke Wälzer konsumiert. Findet sich da was, was die Einschätzung als gewöhnlichen Atheisten rechtfertigt?"

„Nicht, dass ich wüsste. Aber ein ,kritischer ' Realist oder Ontologe setzt natürlich für seine Forschungsarbeit nicht die Existenz Gottes voraus. Das wäre für ihn eine Kompetenzüberschreitung. Insofern ist NH wie dieser Theologe Rudolph ,methodischer Atheist".

„Akzeptiert, Adrian. Während Du die philosophischen Werke NHs studiert hast, habe ich mir seinen Briefwechsel mit seinem Freund aus der Studienzeit in Marburg, Heinz Heimsoeth, der auch Philosophieprofessor wurde, zu Gemüte geführt. Auf diesen Briefwechsel habe ich mich übrigens bei der Erwähnung Bultmanns schon bezogen. NHs zweite Frau Frida schreibt in diesem Briefband, Moment, ich zitiere (S. 320): 
,Nach dem Abitur unternahm er eine größere Wanderung, um zu erproben, ob ihm in der Einsamkeit durch Besinnung auf sich selbst irgend ein Anzeichen von der Existenz Gottes erkennbar würde. Doch mußte er feststellen, daß er sich ständig im Gespräch mit seinen Freunden befand, daß also der Weg des Absehens von allen Beziehungen ihm nicht gangbar und auch nicht überzeugend sein könnte. Er hielt daran fest, daß in der Auseinandersetzung, im Korrigieren des eigenen Denkens an der Meinung des anderen, der wahre Weg der Erkenntnis besteht, und daß die Erkenntnis auf irgendwelche Spuren der Existenz oder des Wirkens Gottes führen müsse, wenn er existiere.

Diese Äußerung über NH von einem Menschen, der ihm nahestand, müsste man schon arg missverstehen, um daraus abzuleiten, er sei Atheist gewesen.“

„O.K., ich habe auch eine Äußerung NHs in „Metaphysik der Erkenntnis“ (in der Folge kurz: ME) 1965, S. 6/7 parat, die dazu passt und die ich Dir mal vorlese:

,Eine rein am Problem orientierte und in diesem Sinne, kritische Ontologie`, wie sie als Grundlage der Gnoseologie anzustreben ist, kann so wenig beweisen, daß es ein ,Erfassen des Ansichseienden' gebe, als sie beweisen kann, daß Gott existiere, Ja, sie kann beides so wenig beweisen als widerlegen. Aber sie kann für das Sein der Erkenntnis, falls es ein solches gibt, sowie für das nicht weniger fragliche Sein ihres Gegenstandes eine gemeinsame Sphäre nachweisen, in welcher beide vergleichbar, vereinbar, ja überhaupt in einer dem Problem genügenden Bezogenheit dastehen.'

Das Zitat führt schon weit über die Aussage hinaus, dass man die Existenz Gottes so wenig beweisen wie widerlegen kann. Besonders wie NH an dieser Stelle fortfährt, wird für unser Gespräch über ihn noch wichtig werden. Jetzt will ich aber erst mal nur noch sagen: Opa, ich glaube ja, Du wolltest mir in unseren Gesprächen über Gott von Anfang an vor allem eines vermitteln: dass die Frage nach der Existenz Gottes nicht mit Antworten wie ,Es gibt ihn“ oder ,Es gibt ihn nicht' beantwortet werden kann, dass ihr wesentlicher Sinn vielmehr darin liegt, den Blick frei für die eigenen Erkenntnismöglichkeiten zu bekommen, die uns für einen angemessenen Umgang mit den Bedingungen unserer Existenz zur Verfügung stehen."

„Vielleicht, ich weiß nicht, da spricht ja schon ziemlich geschliffen ein Philosoph, und jetzt sag nicht ,Opa, verarsch mich nicht!‘. Ich wollte Dir sicher den Kritischen Realismus, die Phänomenologie, die Gestalttheorie und die Gestaltpsychologie der Berliner Schule nahebringen. Meine philosophische Ader nährte sich bis vor kurzem fast ausschließlich von den Schriften der Gestalttheoretiker und -psychologen der Berliner Schule und nachdem ich den Aufsatz geschrieben hatte ,Karl Duncker 'meets' Nicolai Hartmann' bin ich allenfalls auf dem Wege, ein 
Schmalspurphilosoph zu werden. Und auf diesem Wege stelle ich fest, dass alle möglichen Begriffe, derer ich mich naiv bediene, auf total vermintes Territorium führen können."

„Ha, ha, aus dem Zusammenhang schließe ich, dass Du da nicht zuletzt den Begriff, ,Kritischer Realismus' meinst."

„Stimmt. Ich war einmal so naiv anzunehmen, dass der Kritische Realismus, wie ihn die Berliner Schule vertritt, so eindeutig definiert ist, dass er gegen Missinterpretation immun sei, vor allem in der Abgrenzung von Radikalem Konstruktivismus (bzw. Solipsismus) und Naivem Realismus. Aber auch in Abgrenzung von jeglichem Erkenntnistheoretischen Idealismus. Bei NH glaubte ich ihn in weitgehender Übereinstimmung vorzufinden. Dann las ich Sekundärliteratur zu NH; und traute meinen Augen nicht.

Es werden heute Begriffe verwendet, die es seit weit über 2000 Jahren in der Philosophie gibt und die im Verlauf der Philosophiegeschichte in den verschiedensten philosophischen Systemen verwendet wurden und dabei die verschiedensten Bedeutungen annahmen. Verwendet nun heute ein Philosoph einen solchen Begriff, kann er sich wahrscheinlich alle erdenkliche Mühe machen, ihn im Rahmen seiner eigenständigen Systematik neu zu definieren, von früheren Bedeutungen abzugrenzen, die Vergangenheit wird ihn in Gestalt irgendeiner Rezension oder Dissertation einholen. Die Epigonen, von denen $\mathrm{NH}$ immer wieder mal und durchaus nicht ohne jede Wertschätzung spricht, scheinen manchmal wie aus einer irgendwo in der Philosophiegeschichte gelandeten Zeitmaschine zu blicken und von dort über ihre Erinnerung an die Überlegungen eines in der aktuellen Gegenwart eigenständig und originell denkenden Kollegen zu räsonieren, völlig überfordert von dessen neuen, traditionelle Denkfehler überwindenden Gedanken.“

„Opa, Du haust mal wieder - aber das kenne ich ja von Dir - schlusswortartig zu; dabei haben wir doch gerade erst miteinander zu reden begonnen: von Schmalspurphilosoph zu Schmalspurphilosoph; denn so fühle ich mich nach 5 Semestern Philosophie immer noch. Aber richtig an dem, was du sagst, scheint mir auf jeden Fall zu sein: Viele Begriffe der Philosophie sind zu Hülsen geworden, die mit verschiedenen Bedeutungen belegt sind, in Abhängigkeit von erkenntnistheoretischen Standpunkten und umfassenden philosophischen Systemen. Das gilt z.B. für das Wort ,a priori', auf das offenbar kein Philosoph gern verzichtet. Man braucht nur seine spezielle Bedeutung in einem bestimmten philosophischen Gedankengebäude in ein anderes hinein zu behaupten, und schon wird daraus eine Dissertation, die in letzterem Denkfehler nachweist."

„Wie es NH widerfahren ist.“

„Genau, und wir wollten ja über mein Dissertationsthema reden, also darüber, ob und wenn, wo und wie NH und die Berliner Gestalttheoretiker übereinstimmen." 
„Also, dass es Übereinstimmungen gibt, Adrian, habe ich ja wohl schon nachweisen können."

„Ja, ja, Opa, davon hast Du mich mit Deinem Aufsatz überzeugt; genauer gesagt, hast Du mich davon überzeugt, dass Karl Duncker (KD), als Vertreter der Gestalttheoretiker, sich mit Recht in Übereinstimmung mit NH sieht, wenn er die unmittelbare Wahrnehmung eines Baumes z.B. als einen wenn auch noch recht primitiven Akt der Erkenntnis eines Ansichseienden, d.h. eines vom Wahrnehmenden gänzlich unabhängigen Gegenstandes, versteht. Er sieht darin mit NH einen transzendenten Akt, der zu keinem grundsätzlichen Zweifel daran berechtigt, dass Menschen sehen können (wie genau oder ungenau auch immer), was in der absoluten (ontischen) Welt der Dinge tatsächlich existiert. Und das kann jederzeit etwas sein, was der Wahrnehmende nie zuvor gesehen hat. Transzendenz bedeutet hier nichts anderes, als dass die phänomenale Welt des Menschen keine in sich geschlossene Welt ist, sondern offen für das außerhalb und unabhängig von ihr Existierende; noch schärfer gesagt: dass sie sich überhaupt erst im wesentlichen durch die Konfrontation und Auseinandersetzung mit dem, unabhängig von ihr existierenden, Ansichseienden entwickelt."

„Das, Adrian, ist also zunächst einmal - vor aller Betrachtung der in der physikalisch-physiologisch-chemischen Komplexität der Informationsübertragung liegenden Problematik - das übereinstimmende Bekenntnis zu der Auffassung, dass das ,Ding an sich', um das viele Philosophen in Anlehnung an Kant nach wie vor - flapsig gesagt - herumeiern, grundsätzlich menschlicher Erkenntnis (phänomenal) unmittelbar zugänglich ist."

„Ja, jedenfalls wenn wir - zunächst einmal - unter ,Ding an sich' nur das verstehen, was uns über unsere Sinne direkt vermittelt wird. Aber ich würde jetzt gern noch nicht ins Argumentieren für diese Auffassung kommen und auch nicht in die Erörterung ihrer Grenzen und einer eventuellen Anfälligkeit für Verkennung der ansichseienden Realität. Mir erscheint es sinnvoll, mich zunächst einmal so klar wie möglich der erkenntnistheoretischen und ontologischen Auffassungen der Berliner Schule zu vergewissern, als Grundlage für den Vergleich mit NH.“

„Da haben wir ja gerade schon ganz unsystematisch zwei wichtige Punkte angesprochen:

1. Es gibt eine unabhängig von jeglicher Wahrnehmung existierende physische (bzw. ontische) Welt, von Wolfgang Metzger (WM, 1963) als Wirklichkeit im 1. Sinne oder Makrokosmos bezeichnet;

2. Teil dieser Wirklichkeit im 1. Sinne ist auch der Mensch, einschliesslich seiner phänomenalen Welt, von WM als Wirklichkeit im 2. Sinne oder Mikrokosmos bezeichnet. Diese phänomenale Welt..." 
„Halt mal ein, Opa. Du möchtest offenbar alles, was wir eben angesprochen haben, in 2 Punkte packen. Das erscheint mir - darüber habe ich schon nachgedacht - unzweckmäßig. Mach bitte einen 3. Punkt aus dem, was Du jetzt formulieren wolltest."

"Gut.“

„Also

3. Diese phänomenale Welt ist ein komplexes Abbild von Sachverhalten, die die Sinne von der Wirklichkeit im 1. Sinne zum Gehirn übermitteln. In dieser phänomenalen Welt findet sich ein phänomenales Körper-Ich inmitten einer phänomenalen Außenwelt, genauso, wie sich der physische Körper in seiner physischen Umgebung befindet.

4. Das phänomenale Körper-Ich ist bewusstseinsfähig, d.h. es vermag sich seiner selbst und seiner phänomenalen Umwelt bewusst zu werden, vermag Zusammenhänge zu erkennen, planvoll im Rahmen seiner phänomenalen Welt zu handeln und so auch, rückwirkend, seine physischen Existenzbedingungen zu modifizieren."

„Und jetzt muss eine besonders wichtige Auffassung extra als Punkt benannt werden!“

„Ja, mach weiter, Adrian.“

„Gut, also:

5. Das Verhältnis zwischen Original der physischen (Ding-) Welt und unmittelbar gegebenem phänomenalen Abbild wird als ein sehr enges verstanden, das soll heißen: das Abbild ist (im positiven Falle) weitgehend vom Ansichseienden (der ontischen Welt) determiniert. Gerade deshalb ist die (kritische) Erforschung von Irrtümern und sogenannten optischen oder anderen Sinnesorganen zuordenbaren Täuschungen für die Wahrnehmungsforschung so interessant (und fruchtbar) geworden.

Nochmal in schlichten Worten gesagt: Da wir normalerweise keine Schwierigkeiten damit haben, uns in der Welt der uns umgebenden Dinge - z.B. ohne anzustoßen - zurechtzufinden, werden die Ausnahmen zum Gegenstand spezifischer Forschung. Das Ding an sich (ontisches Gebilde) wird in der Regel (gewissermaßen: völlig geistlos) unmittelbar in der Begegnung mit ihm zu einem Ding der phänomenalen Welt, ohne jegliche gedankliche Aktivität des Individuums, ohne dass dieses sich erst mal fragt, was denn dieses oder jenes wohl bedeute und dann erst nach einem Urteil zu sich sagen würde: ,Das halte ich jetzt für einen Baum.‘ Dies ist die Bedeutung der Rede vom unmittelbar Gegebenen." 
„Ja, Adrian, der Mensch braucht nicht einmal ein Wort für etwas zu haben, um $\mathrm{zu}$ wissen, was es ist. Die Frage, wie und warum Sprache entstanden ist, ist eine andere Baustelle, zu der wir ja vielleicht auch noch kommen."

„Ja, vielleicht. Jetzt kann man weitermachen mit

6. Daraus folgt, dass es für die Erforschung der phänomenalen Welten, deren es ja so viele gibt, wie es Individuen gibt, und für die Erforschung der Zusammenhänge zwischen diesen und der ansichseienden Welt nur einen legitimen Ausgangspunkt gibt: die menschliche Erscheinungswelt (die Phänomene), also das, was jemand unmittelbar wahrnimmt. Einen anderen Ausgangspunkt gibt es auch für höhere und höchste Erkenntnis wie die Einsicht in mehr oder weniger komplexe (Gestalt-)Zusammenhänge nicht. (Nur am Rande: Da kann man sich schon fragen, wie treffend eigentlich gängige Unterscheidungen zwischen ,a priori' und ,a posteriori" sind.)"

„Und jetzt wird's heiß, Adrian, oder? Punkt 5 ist Dir schon länger als die vorangehenden Punkte geraten. Punkt 6 wird jetzt, vermute ich, noch viel länger, denn wenn Du jetzt klar sagen willst, was die Berliner Schule unter Phänomenologie versteht, geht es ja schon um Abgrenzung von Brüdern und Schwestern im kritischrealistischen Geiste: Brentano, Husserl, Meinong.“

„Nein, Opa, so lang wird es, jetzt jedenfalls, noch nicht. Ich möchte es jetzt bei der Andeutung der Abgrenzung, die schon in Punkt 5 enthalten ist - der Definition vom unmittelbar Gegebenen, das in statu nascendi von keinem Meinen und Urteilen begleitet wird - belassen, lediglich von einem Zitat Gebrauch machen, das klar und eindeutig und wie ich es nicht kürzer sagen könnte, beschreibt, was die Berliner Schule unter Phänomenologie versteht. Also jetzt

Fortsetzung 6.: Wolfgang Metzger schreibt zum methodischen Vorgehen der Phänomenologie:

,Das Vorgefundene zunächst einfach hinzunehmen, wie es ist; auch wenn es ungewohnt, unerwartet, unlogisch, widersinnig erscheint und unbezweifelten Annahmen oder vertrauten Gedankengängen widerspricht. Die Dinge sprechen zu lassen, ohne Seitenblicke auf Bekanntes, früher Gelerntes, ,Selbstverständliches', auf inhaltliches Wissen, Forderungen der Logik, Voreingenommenheiten des Sprachgebrauchs und Lücken des Wortschatzes. Der Sache mit Liebe und Ehrfurcht gegenüberzutreten, Zweifel und Mißtrauen aber gegebenenfalls zunächst vor allem gegen die Voraussetzungen und Begriffe zu richten, mit denen man das Gegebene bis dahin zu fassen suchte.'

Wohlgemerkt: Metzger spricht davon, wie unmittelbar gegebene Phänomene zur Kenntnis zu nehmen sind - nicht etwa davon, dass man unmittelbar Gegebenes erst einmal von früher Gelerntem, Wissen, Voreingenommenheiten des Sprachgebrauchs etc. 
reinigen müsse, um an reine Phänomene heran zu gelangen. Dieser Unterschied wird später noch eine wichtige Rolle spielen.

7. Als Erkenntnis kann nun eine phänomenale Einsicht bezeichnet werden, die einem ontisch Ansichseienden gerecht wird (der Wahrheit). Ihr liegt immer ein transzendentes Verhältnis zugrunde, d.h. die Teilhabe des phänomenalen Ich an einem von ihm (seinen psychischen Befindlichkeiten) gänzlich unabhängigen Gegenstand oder Geschehen. Erst jenseits dieser Auffassung beginnt für die Berliner Schule die Psychologie im engeren Sinne. Damit distanziert sie sich von jeglichem Psychologismus, aber auch von evolutionstheoretischen Auffassungen, für die logisches Denken und sachliche Gefordertheit rein ontisch-subjektive Lernergebnisse oder dem Überleben geschuldete Denk- und Verhaltensgewohnheiten sind (vgl. z.B. W. Köhler, Werte und Tatsachen, 1968, S. 32.f). Der Kritische Realismus dieser Gestalttheorie führt also zu einer Ontologie, in deren Rahmen die Gestaltbildungsprozesse keine rein ontisch-subjektive (mikrokosmische) Angelegenheit sind, sondern von ontisch-objektiven (makrokosmischen) Gegebenheiten gefordert sein können. Demzufolge vertritt die Berliner Schule als Gestalttheorie eine ihrem Kritischen Realismus immanente Ontologie und als Gestaltpsychologie einen darauf gründenden psychologischen Ansatz der Forschung, der stets das Zusammenspiel psychischer Dynamik und ontischer Gefordertheit kritisch im Auge behält."

„Oh, oh, oh, Adrian, ich hab gebannt zugehört. Aber jetzt hast Du doch eine Menge in Punkt 7 zusammengepackt. Da hätten 3 Punkte draus werden können."

„Schon, Opa, aber das wären dann, abgesehen von der expliziten Abgrenzung zum Psychologismus und zur Überstrapazierung der Evolutionstheorie nur Wiederholungen gewesen. Denn alles andere war schon in den Punkten vorher enthalten."

„Es hat was von einem Schlusswort.“

„Richtig, Opa, das sollte zunächst mal der letzte Punkt für die Beschreibung des erkenntnistheoretischen Ansatzes der Berliner Schule sein.“

„Und der ontologische Ansatz?"

„Der war auch schon enthalten. Der Kritische Realismus führt, konsequent verstanden, eben zu einem differenzierten ontologischen Ansatz und setzt ihn als solchen (durch experimentelle phänomenologische Forschung differenziert im Detail bestätig- oder widerlegbaren) zugleich voraus. Ja, er ist ein ontologischer Ansatz! Darauf werde ich zurückkommen, wenn es um Nicolai Hartmann und den Vergleich mit dessen Auffassungen geht.“

„Einverstanden. Aber müsstest Du jetzt nicht auch kritische Stimmen zu Wort kommen lassen und Dich mit ihnen auseinandersetzen?“ 
„Ach, Opa, das wäre ein weites Feld. Ich will aber doch hauptsächlich die Berliner Schule mit NHs erkenntnistheoretischen und ontologischen Auffassungen vergleichen. Dabei muss ich ja nicht auch noch unbedingt den Wahrheitsbeweis gegenüber allen möglichen kritischen Stimmen antreten. Oder? Ich habe mir vorgenommen, dergleichen nur en passant einfließen zu lassen, wo es sich im Zusammenhang eben anbietet. Aber wo Du das ansprichst, lese ich Dir mal ein paar Metzger-Sätze vor (Hdb.d. Psychologie, 1. Bd, Allgem. Psychologie, 1. Halbbd, S. 15/6):

,Es werden in der Psychologie jedoch auch noch andere [neben naivem und kritischem Realismus] gängige erkenntnistheoretische Standpunkte vertreten. So etwa vertritt v. Uexküll (1921) eine Monadologie im Leibnitzschen Sinne, V. v. Weizsäcker (1940) einen ebenso strengen Kantianismus, nach welchem jede bewußtseinsunabhängige Wirklichkeit grundsätzlich für immer ein allem Erkennen und Vermuten entzogenes X bleibt, und endlich die amerikanische Verhaltenslehre einen strengen Positivismus, z.B. im Sinne Ernst Machs (1922).

Freilich zieht sich durch die ganze Psychologie ein merkwürdiger Widerspruch, indem man zumeist zwei verschiedene Standpunkte einnimmt: einen in den Vor- und Festreden und sonstigen offiziellen Kundgebungen, einen im Alltag der Forschung. Der zweite wird zwar im allgemeinen nicht ausdrücklich formuliert, er ist aber in dem experimentellen Vorgehen und in der Erörterung der Befunde so eindeutig impliziert, daß man ihn unschwer daraus ablesen kann:.....

... kurzum, dieses ganze Vorgehen impliziert die Unterscheidung einer gemeinsamen, bewußtseinsjenseitigen, objektiven Wirklichkeit bzw. physikalischen Welt, eben der Welt der Versuchsanordnungen und sonstigen Reizquellen, von der Menge der davon abgeleiteten Wahrnehmungswelten. Das heißt, es impliziert als Arbeits-Philosophie eindeutig eine Form des kritischen Realismus, der übrigens trotz aller positivistischen Bekenntnisse und Versicherungen, auch die Arbeits-Philosophie des mit seinen speziellen Untersuchungen beschäftigten Physikers ist.

Um der höchst unbefriedigenden wissenschafts-theoretischen Bewußtseinsspaltung in der Psychologie ein Ende zu machen, wird hier vorgeschlagen, die treue und hilfreiche Arbeits- Philosophie auch zur offiziellen Vor- und Festredenphilosophie zu erheben.'

Diese Stellen, Opa, sind im dicken Handbuchband übrigens dick angestrichen und mit Bemerkungen in Deiner Handschrift versehen.“

„Ist wohl Jahrzehnte her. Ich habe mich in den letzten Jahren schon mehrfach gefragt, wo ich bei Metzger explizit zum Begriff kritischer Realismus etwas gelesen hatte. Und kam nicht drauf. Danke für Deinen Fund, Adrian." 
„Übrigens bezieht sich Metzger hier lediglich auf den kleinsten gemeinsamen Nenner aller irgendwie kritisch-realistisch eingestellten Philosophen und Forscher in sonstigen Einzelwissenschaften, wie Du ihn unter Punkt 1 formuliert hast. Schon Punkt 2 ist strittig. Unter sog. Dualisten gibt es wieder solche und solche. Aber das will ich jetzt nicht zum Thema machen."

„Sogar ,radikale Konstruktivisten' denken ja irgendwie kritisch-realistisch gemäß Punkt 1, sonst könnten sie ja nicht behaupten, dass wir über die reale Welt nichts wissen. Sie setzen diese also voraus."

„Ach, Opa, über den Radikalen Konstruktivismus möchte ich am liebsten gar nicht schreiben, denn er ist ja in Wahrheit das krasse Gegenteil einer Erkenntnistheorie; aber ich finde es unvermeidbar, wenn mir klar wird, dass wieder mal jemand radikal-konstruktivistisch kritisiert, ohne sich im entferntesten für einen Radikalen Konstruktivisten zu halten. Das ist immer dann der Fall, wenn ein erkenntnistheoretisch Kritisierender in einer ,Alles-oder-nichts-Argumentationsschleife hängenbleibt. Da muss ich drauf zurückkommen, wenn wir über NH reden.“

Adrian wirkte etwas erschöpft und mir schwirrte der Kopf von allem Möglichen, das man vielleicht noch sagen müsste. Ich sagte aber nur: „Morgen ist auch noch ein Tag, oder?"

„Ja, Opa, jetzt noch ein Pils und einen Grappa. Mein Vater lästert darüber, ich sei ja schon die reinste Wiedergeburt seines Alten.“

„Aber Du rauchst doch nicht. Prost!“

Am nächsten Tag setzten wir, beginnend bei einem über $10 \mathrm{~km}$ langen Spaziergang durch Wald, Wiesen, Täler und über Berge, unser Gespräch fort.

„Ja, wie machen wir denn jetzt weiter, Adrian? Jetzt müssten wir ja wohl nach den Zusammenhängen zwischen allem über Gestalttheorie und Gestaltpsychologie Gesagten und Nicolai Hartmanns (NH) Auffassungen schauen."

„Ach“, murmelte Adrian, „wo anfangen...? Blöderweise bin ich in meinem Kopf jetzt ausgerechnet bei der Replik eines Herrn D’Anna auf Deinen Aufsatz. Die wollte ich bestenfalls in einer Fußnote erwähnen, so überflüssig sie ist. Aber, dann fangen wir halt mit dem Herrn D’Anna an. Wir sind ja unter uns.“

"Ja“, sagte ich, „da hat die Fiorenza Toccafondi in ihrer Eigenschaft als geschäftsführende Herausgeberin der Gestalt Theory, aus welchen Gründen auch immer, wahrscheinlich in bester Absicht, den ,Bock zum Gärtner' gemacht, als sie ihn mit der Bitte ehrte, auf meine Frage zu antworten, wie es komme, dass Hartmann und die erste Generation der Berliner Schule sich gegenseitig nicht namentlich in 
ihren Publikationen erwähnen. Aber natürlich hat er allein die Verantwortung für seinen der Frage nicht gerecht werdenden Artikel. Er hätte ja sagen können: ,Nein, kann ich nicht, weil ich die Berliner Schule nicht kenne und schon deshalb über eventuelle Zusammenhänge mit NH nichts sagen kann'."

„Dazu gehört,Format “, spottete mein inzwischen universitätserfahrener Enkel. „Aber machen wir das kurz. D’Anna drückt sein maßloses Erstaunen darüber aus, dass ein ,Berliner' mit Hartmann einig geht und untermauert sein Erstaunen mit der undifferenzierten Behauptung, Hartmann habe eben der Grazer Schule nahegestanden."

„Das meint, ohne daraus eine Begründung wie D’Anna zu machen, auch Toccafondi."

„Dass jedenfalls, wie D`Anna diese Behauptung strapaziert, Unsinn ist, werden Tatsachen, auf die wir später auf jeden Fall kommen müssen, belegen. In meinem Kopf sind jetzt andere Behauptungen von ihm über $\mathrm{NH}$, auf die einzugehen durchaus eine gute Einleitung zum Zusammenhang zwischen dem ,kritischen Realismus' der Berliner Schule und NHs ,kritischer Ontologie' werden kann."

„Mach mal!“

„Na ja, er erklärt eine Begegnung zwischen den Berliner Gestalttheoretikern und deren ,kritischem Realismus' einerseits und NHs Ontologie andererseits nicht nur deshalb für äußerst unwahrscheinlich, weil NH den ,Grazern' verbunden gewesen sei (die sich im übrigen ja auch als ,kritische Realisten' verstanden), sondern auch, weil er überhaupt kein ,kritischer Realist' gewesen sei.“

„Diese Behauptung hatte mich erst mal richtig beeindruckt und neugierig gemacht, Adrian, weil er ja zugleich zugibt, dass er mit dieser Auffassung allein auf weiter Flur ist, jedenfalls nicht nur der etwas windige Überblicksphilosoph Stegmüller gegen ihn steht. Das fand ich ganz schön mutig."

„Mutig?“, ereiferte sich Adrian. „Ja, selbst mit guten Argumenten sich gegen eine Mehrheit Andersdenkender zu stellen, wäre schon mutig gewesen. Tollkühn aber ist es, eine fragwürdige Behauptung aufzustellen und sich zur weiteren Begründung zu den ausgesprochen irrwitzigen Behauptungen zu versteigen, ,es findet sich bei Hartmann keine haltbare und überzeugende Erkenntnistheorie (D’Anna, Gestalt Theory 1/16, S. 69) und: ,In der Tat hat die kategoriale Ontologie [Hartmanns] mit der Erkenntnistheorie nichts mehr zu tun' (ebd. S. 71)“.

„Ja, starker Tobak von einem, der über NH dissertiert hat, Adrian. Man kann sich wohl fragen, bei welcher philosophischen Sekte jemand, der so etwas behauptet, gelandet sein muss, und zwar ganz egal, ob er damit etwas Positives über $\mathrm{NH}$ sagen will oder etwas Vernichtendes. Das bleibt an dieser Stelle offen..."

„Opa, ich möchte das auch offen lassen. Um nicht nur darüber zu spekulieren, hätte ich mich in die Publikationen D`Annas versenken müssen. Mich interessiert 
derzeit aber neben der Gestalttheorie NH, und dessen Auffassungen glaube ich mittlerweile aus eigener Anschauung beurteilen zu können. NH distanziert sich an keiner Stelle von erkenntnistheoretischen Überlegungen als solchen; er mag sich lediglich mit vielen, wie er sie kennt, nicht identifizieren. Das gilt auch für Varianten des Kritischen Realismus. Mehr kann D’Anna nicht belegen. Selbstverständlich kommt $\mathrm{NH}$ nicht ohne erkenntnistheoretische Reflexion zu seinen eigenen Auffassungen über die Natur menschlicher Erkenntnis und die reale Welt (seiner Ontologie).“

„O.K., Adrian, Du hast D`Anna wahrscheinlich reichlich genug Ehre angetan, indem Du mit ihm unser Gespräch heute eröffnet hast.“

„Genau! Also die Sache ist die,...“ - Ich hing an seinen Lippen. - „dass der Umstand der ist..." Adrian kicherte: „Opa entschuldige den Kalauer. Erst noch ein Zitat, das den Stellenwert der Erkenntnistheorie für NH, wie es an vielen Stellen in allen seinen Werken immer wieder der Fall ist, klarstellt - das übrigens die Fortsetzung der Stelle ist, die ich gestern am Anfang, als wir über Gott sprachen, schon zitiert habe (ME, 1965, S. 7):

,So wenig eine solche Ontologie [gemeint ist die von ihm angestrebte „kritische Ontologie"] jemals der gnoseologischen Bedingtheit enthoben werden könnte - was auch gar nicht in ihren Tendenzen liegt -, so wenig kann auch die Erkenntnistheorie der ontologischen Bedingtheit entwachsen.

Also - jetzt weiter -: Fast immer, wenn NH über Erkenntnistheorie spricht, ist der Begriff, standpunktlich' nicht weit. Wenn man dumm genug ist, kann man daraus folgern, dass er alle erkenntnistheoretischen Überlegungen als ,standpunktlich' ablehnt. Man muss dazu übersehen, dass für ihn eigene erkenntnistheoretische Überlegungen von großer Bedeutung sind. Wie er selbst definiert, bedeutet, standpunktlich ' für ihn, vorurteilsgemäß' (Brief an Heimsoeth v. 30. 6. 1911, zit. auch b. D’Anna, S. 66).

$\mathrm{NH}$ geht es zunächst einmal um einen nicht, standpunktlichen ' Umgang mit dem auch nach Kant ,gemeinsamen Ausgangspunkt aller Erkenntnistheorie'; das ist ,die schlichte Anerkennung eines deutungsfreien Sachverhalts' ,im Erkenntnisphänomen'; das ist ,dieser natürliche und anspruchslose Realismus (ME, s. z.B. IV; 1, 2 und weitere „Einleitung“; 19. Kap. d)).

Dem entgegen steht das, was er ,Korrelativismus' nennt:

,Zum letzteren sind alle Auffassungen zu rechnen, die Erkenntnis und Urteil gleichsetzen, einerlei wie sie sich sonst unterscheiden. So verschiedene Köpfe wie Natorp, Cassirer, Rickert, Husserl, Heidegger sind in dieser Hinsicht demselben Irrtum erlegen. Mit dem Psychologismus aber, den sie bekämpften, ist den logischen Theorien die Verkennung des Transzendenzverhältnisses im Erkenntnisphänomen gemeinsam.... Es gibt kein 
Erkenntnisobjekt ohne Erkenntnissubjekt, sagt dieses [korrelativistische] Argument;...' (Zur Grundlegung der Ontologie, 1965, S. 14; auch zit. v. D’Anna, S. 66).

Nach NH gibt es aber eben Erkenntnisobjekte (Ansichseiendes) auch ohne Erkenntnissubjekt; ein Beleg, des Transzendenzverhältnisses im Erkenntnisphänomen' ist für ihn schon der ohne bewusstes Urteilen auskommende, natürliche und anspruchslose Realismus' des Subjekts; ein Beispiel dafür ist, jetzt mal ganz konkret gesagt, dass jemand, ohne (in der Regel) gegen Laternenpfähle zu rennen oder über Bordsteine zu stolpern, den Alltag bewältigt; erst recht belegen für ihn die ,unbestreitbaren Tatsachen', deren Kenntnis wir dem ,wissenschaftlichen“ Realismus verdanken (z.B. ME, 1965, S. 168) dieses ,Transzendenzverhältnis‘ (vgl. ME, S. 187/8).

Seine Ablehnung des ,logischen Idealismus', demzufolge Gesetze und Relationen nur im Denken bestehen, kleidet er u.a. in die Frage (ME, S. 167): ,Können sie nicht ebenso ursprünglich einem Ansichseienden zugehören?` Eine differenzierte, aber auch etwas längere Antwort NHs u.a. auf diese Frage lese ich jetzt mal vor (ME, S. 187/8):

,Man kann die kritische Ontologie als dritte Stufe einer Entwicklungsreihe auffassen. Die erste ist durch den natürlichen Realismus, die zweite durch die ganze Reihe der spekulativen Standpunkte gekennzeichnet, am ausgeprägtesten durch die idealistischen. Der natürliche Realismus nimmt an, das Reale sei genau so beschaffen, wie das Erkenntnisbild es zeigt. Die spekulativen Standpunkte stellen diese Ansicht auf den Kopf, indem sie zugleich mit der naiv empiristischen Beschaffenheit der Gegenstände auch deren Realität fallen lassen. Sie setzen sie zur bloßen Erscheinung, resp. zum Schein herab. Der Idealismus hebt das in der Objektrichtung gesuchte Reale überhaupt auf.

Die Ontologie nimmt den Mittelweg zwischen diesen Extremen. Ihre These ist die: es gibt ein real Seiendes außerhalb des Bewußtseins, außerhalb der logischen Sphäre und der Grenzen der ratio; die Objekterkenntnis hat Beziehung zu diesem Seienden und gibt ein Stück von ihm wieder, wie sehr immer die Möglichkeit dieser Wiedergabe unbegreifbar sein sollte; aber das Erkenntnisbild deckt sich mit dem Seienden nicht, es ist weder vollständig (adäquat) noch dem Seienden [in seiner physischen Beschaffenheit, der Materie, so denke ich, meint er das ???] ähnlich. Der natürliche Realismus hat recht mit der nackten Realitätsthese, denn das Reale liegt in der Objektrichtung der natürlichen Erkenntnis; aber er hat unrecht mit der Adäquatheitsthese. Die spekulativen Standpunkte haben recht mit der Aufhebung der letzteren, aber unrecht mit der Streichung des Realen aus der Objektrichtung. Die Ontologie verbindet das, worin beide recht haben. Sie behält die Realitätsthese des natürlichen Weltbildes bei, hebt aber die Adäquatheitsthese auf. Sie tut damit etwas Ähnliches, 
wie die wissenschaftliche Weltsicht von jeher getan hat. Sie findet in der Wissenschaft eine ontologische Einstellung vor, welche die Kinderschuhe des naiven Bewußtseins bereits abgestreift hat, und kann sie in gewissen Grenzen zum Ausgangspunkt nehmen. Sie sieht hierbei grundsätzlich von aller standpunktlichen Überspannung der wissenschaftlichen Thesen ab. Mit Materialismus, Energetismus, Biologismus, Evolutionismus hat sie nichts gemein. Sie verschreibt sich nicht der positiven Wissenschaft, tritt nicht in ihren Bann, ist von jeder Art Szientismus und Positivismus gleich weit entfernt wie vom Antiszientismus und Negativismus. Das ,Seiende‘, von dem sie spricht, hat einen viel allgemeineren Charakter. Es geht im Gegenstand der Naturwissenschaft so wenig auf wie in dem der natürlichen Weltsicht. Es umschließt dieses wie jenes und behält noch unbegrenzt Raum für weitere Sphären des Realen.' “

Adrian holte tief Luft und lehnte sich zurück. Ich sagte: „Jetzt mach ich mal den advocatus diaboli: In dieser dreistufigen Entwicklungsreihe kommt der ,natürliche Realismus', der auch ,naiver' genannt wird, vor, aber von ,kritischem Realismus ist gar keine Rede. Fällt der nun für NH unter die ,spekulativen Standpunkte', unter die ,ontologische Einstellung' in der Wissenschaft oder subsumiert er ihn unter der ,Ontologie', wie er sie versteht?"

„Ach, Opa, das ist die Crux, wenn man auf möglichst viel zusammenfassende Abschnitte zum Zitieren erpicht ist. Gut an diesem hier finde ich, dass deutlich wird, worauf NH abzielt. Statt aber Deine Fragen spekulativ zu beantworten, packe ich jetzt den Stier bei den Hörnern mit folgendem Zitat (ME, S. 200):

,In Wirklichkeit aber ist die Sphäre der Ontologie nicht einmal auf diese beiden Richtungen des Transzendenten [Transsubjektives und Transobjektives] beschränkt zu denken, sondern als durchgehende Einheitssphäre des Transzendenten und Immanenten, also sowohl des Objekts als des Transobjektiven, sowohl des Subjekts als des möglichen Transsubjektiven. Damit verliert sie endgültig den Charakter des standpunktlich ausgeprägten Realismus. Sie dürfte sich nicht als ,kritischen` oder ,transzendentalen Realismus' bezeichnen, so nahe der oberflächlichen Betrachtung auch diese Bezeichnungen liegen mögen; dürfte es selbst dann nicht, wenn diese Begriffsprägungen nicht durch anderweitige Systembildung verbraucht und mit Vorurteilen belastet wären.'

Jetzt sehe ich mal von allem, was NH mit standpunktlich' meint, ab, gehe nur auf die Äußerung ein, wonach die Sphäre der Ontologie nicht als ,kritischer ${ }^{6}$ Realismus bezeichnet werden dürfte. Das sieht er so, weil seine konsequent von fragwürdigen Standpunkten befreite kritisch-realistische Analyse des Gegenstandsbewusstseins ihm den Blick frei gemacht hat für das Eindringen in übergegenständliche Eigenschaften der realen Welt. Es geht nicht mehr nur um die Frage, ob das, was Menschen erkennen, real ist, sondern um die Kategorien (und die Schichten) der realen Welt selbst. 
Anders gesagt: NH verwahrt sich implizit dagegen, in seiner ,kritischen Ontologie eine (erweiterte) Erkenntnistheorie zu sehen, und explizit, ,sie von vornherein als Realismus abzustempeln' (ME, S. 199), und das gilt auch dann noch, wenn von ,kritischem' Realismus gesprochen wird, den er zwar in der von ihm von Vorurteilen gereinigten Form vertritt, jedoch nur als ein (,sekundäres', aber unverzichtbares) Fundament seiner Ontologie unter anderen Fundamenten, die sie tragen. Eine besonders wichtige Rolle spielt..."

„Entschuldige, Adrian! Ich bin gerade bei der Frage, die ich immer zu Beginn einer Einführung in den ,kritischen Realismus' der Berliner Schule (der Gestalttheorie), wohl in Anlehnung an Paul Tholey, gestellt habe: ,Wie kommt es, dass wir die Dinge außerhalb von uns sehen, obwohl wir doch wissen, dass sie in unserem Kopf sein müssen, um von uns gesehen zu werden?’ Das ist so, weil die ganze Welt, die wir wahrnehmen, auch in unserem Kopf ist und wir mittendrin sind. Das Draußen, das wir sehen, ist das Draußen im Kopf, keineswegs das Draußen außerhalb unseres Körpers als solches. Grenzen der konkreten Übereinstimmung sind eine Frage der empirischen Erforschung. Die vielen in die Irre führenden Konstruktionen zur Erklärung, wie Dingwahrnehmung entsteht (von Schopenhauers Projektionshypothese bis zur Grazer ,Produktionstheorie') haben das erkennende Ich als Teil der Welt der Dinge in unserem Kopf (bzw. wissenschaftlicher: in unserem sog. Psycho-physischen Niveau = PPN) ausgeklammert oder schlicht übersehen."

„Und eben nachdem das konsequent kritisch-realistisch geklärt ist, wird der Unterschied relevant, wo jemand primär hinwill: zu einer kritisch-realistisch (und ontologisch) fundierten Psychologie oder einer u.a. kritisch-realistisch fundierten Ontologie? Für gute experimentell-psychologische Wahrnehmungsforschung ist kritisch-realistisches Denken - sogar auch, sieht man von sekundären Spekulationen über Untersuchungsergebnisse und daraus folgenden phänomenologischen Schwächen ab, solches mit ,produktions'-theoretischen Vorurteilen - mehr oder weniger hinreichend; für gute Ontologie, wie NH sie versteht, eröffnet aber erst der von diesem und anderen Vorurteilen befreite ,kritische Realismus' die Aussicht, den Ausschnitt der realen Welt, der immanent gegeben ist, als Quelle von Erkenntnis über die Beschaffenheit (Schichten, Sphären, Kategorien) der realen Welt zu nutzen.

Deshalb Phänomenologie! Sie spielt, wie ich eben, als Du mich unterbrachst, fortfahren wollte, für $\mathrm{NH}$ ebenfalls eine (zwar sekundäre, aber unverzichtbare) Schlüsselrolle. Nur gerade nicht so, wie sich das Husserl, Meinong, Benussi (von anderen, die NH nennt, ganz zu schweigen) vorgestellt haben, indem sie dem Individuum nicht in seiner natürlichen Einbettung in das Weltgeschehen begegnen, sondern es als Abstraktum irgendwie außerhalb davon (mehr oder weniger, ideal') konstruiert haben. Es ist eben eine Erkenntnis-Sackgasse, spekulative 
Konstruktionen des Erkenntnisprozesses als Grundlage der Erforschung von Erkenntnis festzuschreiben."

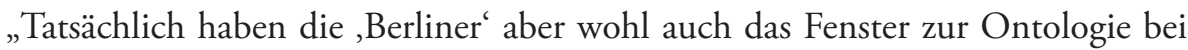
ihren psychologisch-phänomenologischen Untersuchungen schon ziemlich weit geöffnet, indem sie Gestaltgesetze in der Wahrnehmung und im ,produktiven Denken' belegt und beschrieben haben. $\mathrm{NH}$, für den übrigens (was ich nicht unbedingt teile) ,Geistiges Sein... keine psychologische Angelegenheit‘ ist („Das Problem des geistigen Seins“, in der Folge kurz: GS, S. 48), bemerkt in einer Fußnote dazu (GS, S. 49):

,Daß die Psychologie selbst sich seitdem in manchen führenden Vertretern umgestaltet hat und zu einer Wissenschaft vom Geist geworden ist, soll hier keineswegs verkannt werden. Nur eben ist sie insofern mehr als ihr Name besagt, und sollte sich vielleicht auch anders nennen“ (s.a. „Der Aufbau der realen Welt", in der Folge kurz: RW, S. 137).

Wen genau er damit meint, läßt NH offen; es kämen schon mehrere Zeitgenossen, abgesehen von Wertheimer, Köhler, Koffka u.a. in Frage. Aber belegen kann ich, was NH über Karl Dunckers (KD) Habilitationsschrift ,Zur Psychologie des produktiven Denkens' exklusiv, nämlich in einer handschriftlichen gutachterlichen Äußerung (o.D.; 1935?) schreibt. Das gehört, scheint mir, unbedingt hierher:

„Prof. Nicolai Hartmann, Berlin-Neubabelsberg

Grossbeerenstrasse 302

Dunckers Arbeit ist durch ihre neuartige Methode außerordentlich lehrreich. Sie ist in erkenntnistheoretischer Hinsicht vielleicht noch aufschlußreicher als in psychologischer, indem sie mitten aus der Praxis des vor bestimmte Aufgaben gestellten Denkens die eigenartigen Wege aufdeckt, die es beim Finden der Lösung einschlägt. Man sieht den lebendigen Gedanken an der Arbeit, wie er suchend und tastend ins Unbekannte vorfühlt, und man erfährt sehr genau, was über sein Gelingen oder Versagen entscheidet.

Nicolai Hartmann“ “

„Ja, Opa, in diesem Gutachten bestätigt er $\mathrm{KD}$, dass der einen lehrreichen empirischen Beitrag zum geistigen Sein und - wohl ohne den letzten Satz über zu interpretieren - auch zur Frage der Übereinstimmung von Erkenntnis-Kategorien und Seins-Kategorien beigesteuert hat. Damit spricht er, so scheint mir, der phänomenologisch-experimentellen Forschung der Berliner (denn auf deren Forschungen gründet KDs Arbeit) einen Platz im Rahmen seiner ,kritischen Ontologie‘ zu, spezieller gesagt: im Rahmen seiner Schichtenlehre 
(physische, organische, psychische und geistige Schicht) und seiner Kategorialanalyse (von den jeweiligen Seinskategorien der Schichten bis zu den Fundamentalkategorien).“

„Ja, ja, das kann man wohl so sehen. Im übrigen könnte KDs Arbeit ein Anlass für seine Rede davon gewesen sein, die Psychologie habe sich zum Teil schon umgestaltet zu einer Wissenschaft vom Geist (s. Zitat oben). Aber KD weist im Vorwort seiner „Psychologie des Produktiven Denkens“ (S. III) auch ausdrücklich darauf hin, dass er

,mit einer gewissen brutalen Konsequenz' ,bei den denkpsychologischen Analysen bewußt abgesehen' habe, von allen Sachverhalten, die für das Problem des Findens... nicht unmittelbare Bedeutung haben - mögen sie auch noch so wesentlich zum vollen psychologischen Bestand eines Findungsprozesses gehören'.

Damit meint er, unter Verweis auf Kurt Lewin, ihr Einhergehen mit im engeren Sinne psychischen Erscheinungen wie Bedürfnisse und Affekte, z.B. Interesse, Erfolgs- und Misserfolgserlebnisse, Ersatzhandlungen, Resignation und Ärger oder Leistungsstolz.

Wahrnehmen und (Nach-)Denken findet stets im Kontext von im engeren Sinne psychischen Befindlichkeiten wie Wünschen und Hoffen, Bedürfnissen, Streben, Vorstellungen, Erwartungen, Neigungen statt. Dunckers Lehrer Max Wertheimer zeigt das sehr anschaulich in seinem Buch „Produktives Denken“ in den Kapiteln „Zwei Jungen spielen Federball“ und „Ein Mädchen beschreibt sein Büro“. Und Metzger zeigt in "Schöpferische Freiheit“, wie eigensüchtige Nebenziele ,den Zug des Ziels` (einer sachlichen ,Gefordertheit‘ bzw. ,Forderung der Lage') beeinträchtigen oder ganz zum Verschwinden bringen können. Hierin ist impliziert, dass der Mensch prinzipiell in der Lage ist, zwischen einem „Befindlichkeits-Bewusstsein` (z.B. bzgl. Gefühlen und Strebungen; vgl. Metzger, 1966, S. 5) und sachlicher Gefordertheit (dem u.a. im Sinne NHs Geistigen) zu unterscheiden vermag. Wenn er das nicht könnte, hätte KD seine ,denkpsychologischen' Untersuchungen gar nicht durchführen können.

In der gestalttheoretisch-phänomenologischen Forschung spielt aus Gründen der methodischen Zugänglichkeit die Erforschung von Realkategorien der Wahrnehmung am Beispiel physikalisch und mathematisch definierbarer Gegenstände, in der Mehrzahl figuraler Gebilde, eine bedeutende Rolle. In diesem Bereich konnten ,Ontogramm' und ,Phänogramm' (vgl. zur Terminologie Rausch, 1966, S. 780-803) für die Untersuchung geometrisch-optischer Täuschungen präzise miteinander verglichen werden. Dieses Vorgehen steht im Einklang mit NHs wohlbegründeter Überzeugung, dass zwischen den physikalischen Erkenntniskategorien und den Kategorien der Seinsschicht des Anorganischen große und 
zwischen den mathematischen und den Seins-Kategorien des Quantitativen fast totale Übereinstimmung besteht (NH, 1949, S. 148/9).“

„Dein Vortrag wird lang, Opa. Ich ahne, was Dich antreibt, so weit auszuholen, nachdem ich selbst so tollkühn war, NHs Kategorialanalyse explizit ins Spiel zu bringen. Ich möchte abkürzen. Bieten die Berliner erwähnenswerte Fortschritte im Sinne von NHs Überlegung (RW, S. 136/7):

,Da nun aber zwischen den Akten [des physisch realen Lebens] und den objektiven Inhalten des Bewußtseins bei aller Verschiedenheit doch ein unverkennbar durchgehender Zusammenhang besteht, so muß es auch hier eine modifizierte Wiederkehr gewisser Kategorien geben. Die Aufgabe, diese herauszuarbeiten, sowie in ihr das entsprechende Verhältnis von Identität und Nichtidentität im Verhältnis zu den übrigen Sphären klarzustellen, dürfte der Psychologie angehören. Freilich würde das eine Umbildung der Psychologie von Grund aus bedeuten; es läßt sich aber nicht verkennen, daß die heutige Psychologie in dieser Umbildung bereits begriffen ist, sofern es ihr nicht mehr um letzte Elemente, sondern um Gestalten und Ganzheiten, also um solche Gebilde geht, die deutlich kategorialen Charakter zeigen.?"

„Dieser Aufgabe, Adrian, haben sich die Berliner nun doch recht erfolgreich gestellt..."

„Und zwar schon, bevor NH 1939 zu Beginn des auf den zitierten Absatz folgenden Absatzes schreibt:,Für die Belange einer eigentlich ontologischen Psychologie, verstanden als Erforschung des seelischen Seins, mag es heute noch nicht an der Zeit sein, sie in Angriff zu nehmen.' Erst NH hat mich darauf gebracht, dass es vielleicht gar nicht so falsch wäre, gestalttheoretische Psychologie als ,ontologische Psychologie' zu apostrophieren. Ausgehend von einer hochdifferenzierten experimentell-phänomenologischen Analyse der Realkategorien in der Wahrnehmung und des einsichtigen Denkens haben die Berliner das ,Prägnanzgesetz (auch ,Gesetz der guten Gestalt') als durchgehende Kategorie zumindest des Psychischen und des Geistigen erwiesen. Darunter fallen die Gestaltgesetze bzw. Faktoren: 1. ,Gleichartigkeit' (Gleichheit und Ähnlichkeit) und der ,geringsten Inhomogenität‘; 2. der ,Nähe' und der ,größten Dichte‘; 3. des ,gemeinsamen Schicksals‘ (übereinstimmendes Verhalten); 4. der ,(objektiven) Einstellung;; 5. des ,Aufgehens ohne Rest‘; 6. der ,durchgehenden Kurve‘ (des glatten Verlaufs); 7. der, Geschlossenheit‘ (vgl. Metzger, u.a. 1966, S. 699 ff.). Die weitere differenzierte Auseinandersetzung mit dem ,Eigenschaftsproblem in der Gestalttheorie der Wahrnehmung' (Rausch, 1966, S. 866-953, s. auch Metzger, 1963, 2. Kap., S. 48-95) führt zur Definition von Prägnanzkriterien, Kriterien für Prägnanzstufen und Gestalthöhe. Dass es sich auch dabei um Realkategorien des Psychischen (einschließlich des Geistes) handelt, ist für die Autoren unzweifelhaft. 
Und zumindest einen der durchgehenden, Seinsgegensätze ${ }^{‘}$ NHs scheinen sie mir empirisch überzeugend bestätigt zu haben, nämlich ,Substrat - Relation'; ich glaube aber, dass sich, mal nur implizit, mal mehr oder weniger explizit in ihren Erörterungen viel Übereinstimmung auch mit den anderen, Seinsgegensätzen' bei NH findet. Für die Übereinstimmung im Detail lege ich angesichts der nach eigener Einschätzung NHs manchmal eher kompliziert erscheinenden Begründungen seiner, Gegensätze (obwohl sie mir schließlich alle einzuleuchten schienen), derzeit aber meine Hand noch nicht ins Feuer. Für die Überprüfung im Detail wäre etwas größerer Aufwand vonnöten, nicht so sehr auf der Seite von $\mathrm{NH}$, umso mehr aber auf der Seite der Berliner, weil es ihnen ja nicht um eine systematische Auflistung von Fundamentalkategorien geht; sie erörtern solche Gesichtspunkte zumeist nur am Rande im Zusammenhang mit jeweils aktuellen Forschungsexperimenten. Man müsste deshalb Stelle für Stelle z.B. in Metzgers ,Psychologie` aufsuchen, wo die von NH verwendeten Begriffe vorkommen und prüfen, ob und wieweit sie übereinstimmend verstanden werden.“

„Nun, Adrian, es wäre wirklich nicht verwunderlich, wenn da zumindest scheinbare Unstimmigkeiten aufträten, denn unsere Sprache ist ja kein besonders präzises Denkinstrument. Jedenfalls glaube ich auch, dass das Gegensatzpaar ,Substrat Relation' - von dem NH sagt (RW, S. 255), dass es sich nur einseitig abstufen lässt, nämlich auf der Seite der ,Relation ' - bei den Berlinern eine besondere Rolle spielt."

„Ja, da ist die Übereinstimmung zwischen NH und den Berlinern augenfällig. Die folgenden Sätze NHs (RW, S. 255) lassen sich problemlos mit Metzgers ,Gestalttheorie des Zusammenhangs'vereinbaren:

,Es zeigt sich, daß der innere Bau der sog. Dinge selbst ein relationaler ist; Relationen also sind bereits Aufbaumomente in ihnen, denn aller Aufbau ist Zusammenhang, Relation aber ist nichts anderes als das kategoriale Schema des Zusammenhangs als solchen.'

In Metzgers ,Psychologie` trägt das 3. Kapitel den Titel ,Das Problem des Zusammenhangs' (1963, S. 96-130). Hier erläutert Metzger in 6 Kernsätzen u.a., dass der ,Zusammenhang' von Ganzen und Gruppengebilden sich nur von Gestalteigenschaften her verstehen lässt. Demzufolge müsste es sich bei der Gestaltwahrnehmung um eine Erkenntniskategorie handeln, die durch alle Seinsschichten hindurch ihre Identität wahrt."

„Ich glaube, lieber Adrian, dass wir gerade einen Anfang für einen weit über Grundlagen hinausgehenden Vergleich zwischen NHs kritischer Ontologie und der Gestalttheorie und -psychologie der Berliner skizziert haben. Ich fasse das jetzt mal als vorweggenommenen Ausblick auf, der eigentlich an den Schluss Deiner Dissertation gehörte." 
„Ja, gut, Opa, jetzt sind wir, reichlich unsystematisch, schon ins Vergleichen zwischen NH und Berlinern gekommen. Ich würde gern geordneter vorgehen, also vor dem systematischen Vergleich der Grundlagen möglichst alles dafür über NH Wichtige schon mal gesagt haben."

„Ja, natürlich, was also fehlt da noch? Ach so, die angebliche Nähe NHs zur Grazer Schule, insbesondere zu Meinong. Darf ich mal loslegen?“

„Bitteschön, Opa! Mit ,angeblich` hast Du ja den Tenor Deiner Stellungnahme schon angekündigt."

„Ach so, ja. Also, Du hast mir ja schon in einem Brief gesagt, dass Du mich, zumindest an einer Stelle, bremsen wirst. Tu das. Ich lege mal mit meiner ursprünglichen Auffassung los. Für mich war, nachdem Toccafondi mir zum ersten Mal (ohne weitere Erläuterung) geschrieben hatte, NH sei der Grazer Schule verbunden gewesen, noch die Hauptfrage, ob sich irgendwo bei ihm eine Nähe zur ,produktionstheoretischen' Gegenstands-Auffassung im Grazer ,kritischen Realismus' erkennen ließe. Das konnte ich mit überwältigend zahlreichen Stellen in fast allen Hauptwerken widerlegen."

„Ja, Opa, wer daran zweifelt, kann NH nicht kennen. Vielleicht am deutlichsten wird das in dem, was er in der Diskussion zu seinem Vortrag ,Zum Problem der Realitätsgegebenheit 1931 sagt, indem er nämlich unmissverständlich feststellt, dass ihm am Husserlschen ,Aktschema' nichts liegt. Hier nennt er endlich mal Roß und Reiter.“

„Und dort fortfährt, Adrian, ich zitiere:

,Die beliebte Vorstellungsweise, als wäre das Subjekt zuerst einmal in sich gefangen und müßte dann erst ausbrechen, um ein Realitätsbewußtsein zu gewinnen - eine Ansicht, die mir heute von mehr als einer Seite nachgesagt wurde -, ist die meinige nicht. Es gibt kein wirkliches Bewußtsein, das nicht von vornherein aufgeschlossen im Zuge der Realgeschehnisse drinstände' (in Hartung \& Wunsch, Hg., S. 258/9).

Wie das Zitat belegt, erlebt sich NH in seiner Auffassung, dass es sich bei der unmittelbaren Gegenstandswahrnehmung um einen ,transzendenten Akt ' handelt, hartnäckig missverstanden."

Adrian seufzte: „Ja, das zeigt, wie unvorstellbar es für viele Philosophen damals war (und vielleicht heute noch ist), die von Brentano (trotz all seiner Verdienste, vgl. Stegmüller, 1975, S. 2-48) gelegte Spur eines noch teilweise idealistischen ,kritischen Realismus' (vgl. z.B. Zitat bei Duncker, in Boege \& Walter, Hg., 2008, S. 42, auch in Walter, 2016, S. 8, Fußn. 5, 2.) zu verlassen, der zufolge generell ein Urteil (und sei es ein unbelegbar unbewusstes) Voraussetzung für Erkenntnis ist. Das ist für NH der Musterfall von ,standpunktlich' (= vorurteilsgemäß), wie 
er nicht müde wird, immer wieder zu betonen. Im übrigen habe ich mich aber gefragt, warum er hier nicht auch Meinong nennt."

„Denn da ist auch nicht dran zu zweifeln“, fiel ich ein, „dass Meinong in diesem Punkt im wesentlichen mit Brentano und Husserl einig geht, so sehr er auch mit letzterem, fast bösartig, konkurriert, wie sein Briefwechsel mit NH offenbart (vgl. Philosophenbriefe). Aber, ja, warum nennt er nicht Meinong? Irgendwo schreibt NH einmal - ich weiß nicht mehr wo, sage es deshalb mit meinen Worten -, er kenne das Gefühl einer ,Beißhemmung', das ihn gelegentlich hindere, scharfe Kritik zu äußern."

„Ich wüsste im Falle Meinongs einen Grund“, warf Adrian ein, „aber sag Du mal".

„Na ja, der briefliche Kontakt zwischen NH und Meinong wurde von einer von Meinong offenbar sehr geschätzten Schülerin vermittelt (vgl. Meinong, 1965, Philosophenbriefe), die zu den ,Neukantianern' (Cohen und Natorp) nach Marburg gewechselt hatte, wo auch NH studierte."

„Ja, weiß ich, den Briefwechsel NH-Meinong habe ich ja auch gelesen. Und?“

„Und..., also die Briefe habe ich mir ja erst besorgt, nachdem Toccafondi mich in einem weiteren Brief auf sie hingewiesen hatte als Beleg für die persönliche und sachliche Nähe zwischen NH und Meinong. Ich denke, dass es für NHs seelisches Gleichgewicht nicht unwichtig war, mit einem hochangesehenen und -geehrten Philosophieprofessor korrespondieren zu können, während er, noch blutjung, im 1. Weltkrieg u.a. in Russland eingesetzt war, zumal er sich da von seinen Lehrern in Marburg schon sehr distanziert hatte. Ein weiterer Grund ach wir sind ja unter uns - könnte gewesen sein, dass sich zwischen NH und der erwähnten Meinong-Schülerin eine enge persönliche Beziehung entwickelte, die man wohl ganz ohne Mutmaßung (vgl. Briefwechsel mit Heimsoeth, zwischen 1916 und 1918) als ,intime ' bezeichnen darf."

„Oh, da habe ich wohl den Briefwechsel mit Heimsoeth etwas zu oberflächlich gelesen. Die Stellen, Opa, musst Du mir zeigen.“

„Später. Jetzt erst noch mal zur Frage, ob man wirklich von großer Nähe zwischen $\mathrm{NH}$ und Meinong sprechen kann. Wie ich schon sagte, meint Toccafondi unter Verweis auf den Band (1965) ,Philosophenbriefe. Aus der wiss. Korrespondenz von Alexius Meinong', dass eine besondere Nähe zwischen den Auffassungen von $\mathrm{NH}$ und der Grazer Schule der Gestaltpsychologie bestanden hätte. Dort findet sich auf den Seiten 208-220 der von 1915-1920 währende Briefwechsel zwischen $\mathrm{NH}$ und A. Meinong.

Große Verbundenheit bestätigt der Briefwechsel aber keineswegs. Was die Einschätzung persönlicher Verbundenheit anbelangt, so können die dem deutschen 
Kaiserreich geschuldeten höfisch-höflichen Floskeln wechselseitiger Verehrung sehr in die Irre führen. Aus ihnen kann man allenfalls ableiten, dass hier ein in den Anfängen seiner akademischen Laufbahn stehender und ein auf dem Höhepunkt seines akademischen Ansehens angekommener Philosoph miteinander zu kommunizieren versuchen, ohne sich, wo sie divergierende Auffassungen und Zielsetzungen anklingen lassen, gegenseitig allzu sehr zu verletzen. In der Sache ist die Kritik NHs (z.B. im längsten der zitierten Briefe vom 26. 1. 1919) an Meinongs ,Beweisen' für ein allgemeines Kausalgesetz geradezu unerbittlich, und Meinong weicht zuvor schon in seinen Antworten einem wirklichen Eingehen auf NHs Auffassungen fast durchgehend mit vielen Ausflüchten (äußere Umstände, Zeitmangel) aus.

Beide sind im Zusammenhang mit der „Frage der Beweisbarkeit des Kausalgesetzes“ interessiert am ,Modalproblem' (Möglichkeit, Wirklichkeit, Notwendigkeit). Dieses Thema, das beide verbindet, trennt sie in Behandlung und Zielsetzung aber auch entschieden voneinander. Wenn man es böse sagen will, kann man behaupten, dass NHs Interesse an Meinong vor allem darin sich zuspitzt, ihm beim Scheitern des Versuchs, ein ,allgemeines Kausalgesetz' modal zu erweisen, gespannt über die Schulter zu sehen und aus den Details dieses Scheiterns Argumente für die Bestätigung seiner eigenen Auffassung abzuleiten, der zufolge es sich in der Allgemeinheit, in der Meinong es erweisen möchte, gar nicht erweisen lässt.

Gut, Adrian, ich lasse es mal dabei, weil ich ja schon weiß, dass Du das etwas anders siehst."

„Ach, so sehr anders als Du sehe ich das gar nicht. Aber man kann die Frage der Verbundenheit, der in der Sache jedenfalls, auch etwas anders bewerten, wenn man in dem von Dir schon erwähnten Brief NHs (vom 26. 1. 1919) liest:

,Ich möchte gleich zu Anfang gestehen, dass mich seit langer Zeit kein Buch auf dem Gebiet theoretischer Spekulation so gefesselt hat wie dieses. Sie wissen, daß ich im Gegensatz sowohl zur Schule Natorps als auch zur Schule Husserls mich um die Gewinnung einer ontologischen Basis bemühe. Dass mir aber in diesem Bemühen die ,Gegenstandstheorie'so weit entgegenkommt, habe ich nach Ihren früheren Veröffentlichungen doch nicht annähernd erfaßt. Als ich vor 4 Jahren einen kleinen Versuch über das Seinsverhältnis der Modalitäten veröffentlichte, da war mir die freundliche Zustimmung, die ich gerade von Ihrer Seite erfuhr, in ihren inneren Motiven keineswegs durchsichtig. Heute glaube ich klarer zu sehen, und das verdanke ich Ihrer Schrift über das Causalgesetz. Was mir nämlich nicht in den Sinn gekommen war, die Modalanalyse direkt für ein in meinem Sinne, ontologisches' Problem fruchtbar zu machen, das haben Sie nunmehr wahr gemacht. Dass aber der Gegenstand dieser Anwendung gerade das Causalgesetz ist, giebt mir noch ein zweites Interesse an der 
Sache, weil mir selbst die Bemühung um die Beweisbarkeit desselben aus vielfachen Gründen naheliegt.'

Zumindest dieses spezielle Interesse an der, Modalanalyse verbindet beide. Und es klingt für mich auch glaubhaft, wenn NH sagt, dass ihn Meinongs ,Schrift über das Causalgesetz' erst darauf gebracht hat, ,die Modalanalyse für ein in meinem Sinne 'ontologisches' Problem fruchtbar zu machen'. Und ebenso verbindet sie der Briefstelle zufolge die Beschäftigung mit dem ,Kausalgesetz‘. Also, wenn ich von mir ausgehe, kann ich sagen, dass ich wahrscheinlich schon eine gewisse Dankbarkeit jemandem gegenüber spüren würde, durch dessen Arbeit ich bei meiner eigenen weiterkomme."

„Wenn aber nun“, bohrte ich nach, „jemand allgemein behaupten würde, NH sei von Meinong beeinflusst worden - ein Herr Morgenstern behauptet das, glaube ich -, würdest Du da zustimmen?“

„In dem von NH zum Ausdruck gebrachten Sinne ja, aber nein - ich nehme an, darauf willst Du hinaus -, wenn damit gemeint sein soll, NH sei Schüler Meinongs gewesen. Dazu wusste NH schon vor seinem Briefkontakt mit Meinong viel zu genau, worauf er hinauswollte. Dafür spielte die kritische Auseinandersetzung mit seinen neukantianischen Lehrern Cohen und Natorp in Verbindung mit seiner enormen Kenntnis der Philosophiegeschichte eine unvergleichlich größere Rolle."

„Und was ist mit Meinongs, Gegenstandstheorie‘?" Ich ließ nicht locker.

„Damit hatte er ,nichts am Hut'.“

„Ich bin beruhigt, Adrian. Das geht ja auch aus dem Briefzitat mehr oder weniger hervor."

„Ja, so lässt sich verstehen, wenn NH in seiner Rezension von Meinongs Kausalarbeit schreibt:

,Im Interesse der Kürze und Gemeinverständlichkeit sei es gestattet, von der aufs feinste herausgearbeiteten 'gegenstandstheoretischen' Einkleidung des [modalen] Arguments abzusehen und es in freier, vereinfachter und terminologisch unverbindlicher Form wiederzugeben' $(\mathrm{NH}, 1919$ zuerst ersch. in „Kant-Studien, Bd. XXIV, Heft 3, S. 261-290, zit. nach Kleinere Schriften III (1958, S. 246).

Im übrigen findet sich an manchen Stellen in NHs Werk - ganz abgesehen von der Kritik an der ,produktionstheoretischen' Annahme - implizite und explizite Kritik am ,gegenstandstheoretischen' Denken Meinongs, unter Namensnennung nur selten, aber z.B. im Aufsatz ,Heinrich Maiers Beitrag zum Problem der Kategorien` von 1938 stimmt er diesem darin zu, einem ,gegenständlichen Apriorismus, etwa nach der Art, wie Meinong und Husserl ihn durchzuführen 
versucht haben', nicht zu folgen, demzufolge ,die Kategorien am reinen Wesen des Gegenstandes, im Absehen von dessen empirischer Besonderheit, unmittelbar einsichtig sein' müssten (NH, zuerst veröffentlicht 1938, zit. nach Kleinere Schriften Bd. II (1957), S. 350).

Um aber Meinong nicht Unrecht zu tun, möchte ich doch noch seinen letzten Brief an NH erwähnen (Philosophenbriefe, S. 219/20; das Datum 8. 2. 1920 mag stimmen, dann stimmt allerdings keinesfalls das im Antwortbrief NHs angegeben Datum, nämlich 6. 2. 1920). Mir scheint, dass er darin ehrlich bedauert, ein eingehendes Studium von NHs Arbeiten, versäumt' zu haben. Was für eine, andersartige Hauptarbeit' ihn, die eigentliche Verarbeitung des von Ihnen Gebotenen auf eine hoffentlich nicht allzu ferne minder stark in Anspruch genommene Zeit aufsparen' lässt, teilt er allerdings nicht mit. Vielleicht hat er wirklich vorgehabt, sich mit NHs Arbeiten eingehend zu beschäftigen. Sollte es so gewesen sein, so hat dies jedenfalls sein Tod im Herbst desselben Jahres vereitelt."

„So und jetzt erklär mir noch schnell detailliert die Meinongsche ,Gegenstandstheorie!

Adrian schreckte auf: „Opa, spinnst Du? Ach so“, fügte er ausatmend hinzu, nachdem er mir ins Gesicht geschaut hatte, und lachte: „Das hätte noch gefehlt. Ich hab ja tatsächlich seine Kausalarbeit und spezielle Texte von ihm über ,Gegenstandstheorie ' gelesen, in denen er sie stolz als eine neue eigenständige Wissenschaft bezeichnet. Dann auch noch in eine Dissertation darüber hineingeschaut (Chrudzimski, 2007). Das hat mir gereicht. Für meine Dissertation brauche ich seine komplizierte ,Gegenstandstheorie‘ nicht. Vielleicht muss ich mich mit der Modalitätsfrage aber noch mehr auseinandersetzen, als ich es bisher getan habe. Aber dazu findet sich hinreichendes Material bei NH. Noch nicht ganz spruchreif ist mein Eindruck, dass es sich lohnen könnte, nach Übereinstimmungen zwischen W. Köhlers Analyse von ,Gefordertheit und ,Kräften' in seinem Werk, Werte und Tatsachen' und NHs Modalanalyse zu schauen."

[Der Beitrag wurde an dieser Stelle auf Grund redaktioneller Vereinbarungen geteilt. Seine Fortsetzung bis zu seinem Schluss folgt in der nächsten Ausgabe der "Gestalt Theory" (Heft 1/2019).]

\section{Zusammenfassung}

Der Beitrag belegt die Übereinstimmung in wesentlichen Grundlagen zwischen der Berliner Schule der Gestaltpsychologie (Gestalttheorie) und Nicolai Hartmanns Kritischer Ontologie. Diese Übereinstimmung manifestiert sich u.a.

a) in der Bedeutung, die der Erkenntnistheorie in der Ausprägung eines von idealistischen (u.a. produktionstheoretischen) Vorurteilen gereinigten Kritischen 
Realismus im Zusammenwirken mit einer vorbehaltlosen Phänomenologie des Gegebenen beigemessen wird;

b) in der daraus hervorgehenden Auffassung, dass der Mensch (realer) Teil der ansichseienden Welt ist und es „kein wirkliches Bewußtsein“ gibt, „das nicht von vornherein aufgeschlossen im Zuge der Realgeschehnisse drinstände“ ( $\mathrm{NH})$.

c) in der ebenfalls so gewonnenen Auffassung, dass Dingerkenntnis keines phänomenologisch unbelegbaren subjektiven „Urteils“ bedarf, vielmehr das Ergebnis eines „transzendenten Aktes“ ist, der prinzipiell den Zugang zur Erkenntnis des Ansichseienden („Ding an sich“) ermöglicht;

d) in der Auffassung, dass die Frage der Angemessenheit von Dingerkenntnis (Spezialfall: geometrisch-optische Täuschungen) empirisch (phänomenologischexperimentell) geklärt werden kann. Hierbei spielt die erfahrungsgemäß hohe Übereinstimmung von physikalischen und mathematischen Erkenntniskategorien mit den Seinskategorien des Anorganischen und des Quantitativen eine entscheidende Rolle (ermöglicht Vergleich von „Ontogramm“ und „Phänogramm“).

Schon aus den (hier) ausgewählten Übereinstimmungen ergibt sich eine gemeinsame kritische Distanz zu Brentano-Schülern wie Husserl und Meinong und zum Neu-Kantianismus.

Schlüsselwörter: Nicolai Hartmann, Berliner Schule der Gestaltpsychologie (Gestalttheorie), Kritische Ontologie, Kritischer Realismus, Phänomenologie, transzendenter Akt, Produktionstheorie, Fundierungstheorie, Grazer Schule der Gestaltpsychologie.

\section{Nicolai Hartmann's Critical Ontology and the Critical Realism of the Berlin School of Gestalt Psychology}

\section{A Fictional Dialogue About an Unwritten Dissertation}

\section{Summary}

The author exemplifies the congruency of essential foundations between the critical realism of the Berlin School of Gestalt Psychology (Gestalt theory) and Nicolai Hartmann`s Critical Ontology. For instance, this congruency manifests in the importance given to critical-realistic epistemology - purified from idealistic prejudices, not least prejudices such as production-theoretical ones - connected with an unconditional phenomenology. Altogether, it results in a shared critical distance from scholars of Brentano, such as Husserl and Meinong, as well as from Neo-Kantianism.

Keywords: Nicolai Hartmann, Berlin School of Gestalt Psychology (Gestalt theory), Critical ontology, Critical realism, Phenomenology, Transcendental act, Production theory, Graz School of Gestalt Psychology.

\section{Literatur}

Arnheim, R. (1960). Gestalten von gestern und heute. In F. Weinhandl (Hrsg.).(1974³): Gestalthaftes Sehen. Zum hundertjährigen Geburtstag von Christian von Ehrenfels (S. 79-85).Darmstadt: Wissenschaftl. Buchgesellsch 
Barth, K. (1938).Dogmatik. Bd. I, 2. Halbbd.: Die Lehre vom Wort Gottes. Zollikon: Verlag der Evangelischen Buchhandlung.

Boege, H. \& Walter, H.-J. P. (Hrsg.).(2008).Karl Duncker, Erscheinung und Erkenntnis des Menschlichen. Wien: Krammer.

Boudewijnse, G.J. (1999). The rise and fall of the Graz School. Gestalt Theory 21, 2, 140-158.

Chrudzimski, A. (2007). Gegenstandstheorie und Theorie der Intentionalität bei Alexius Meinong. Berlin: Springer.

D’Anna, G. (2016). Nicolai Hartmann und Gestalttheoretische Psychologie: Ein unwahrscheinliches Treffen (Diskussionsbeitrag). Gestalt Theory 38, 1, 65-74.

Duncker, K. (englisch: 1939, deutsch: 2003). Ethische Relativität? In H. Boege \& H.-J.P.Walter (Hrsg.).(2008), 64-80.

Duncker, K. (englisch: 1941/42, deutsch: 2002). Über Lust, Emotion und Streben. In H. Boege \& H.-J.P. Walter (Hrsg.).(2008), 81-118.

Duncker, K. (verfasst um 1935). Zur Kritik der materialistischen Geschichtsauffassung. In H. Boege \& H.-J.P. Walter (Hrsg.).(2008), 119-142.

Duncker, K. (verfasst 1935) Erscheinungslehre und Erkenntnistheorie des Gegenstandsbewußtseins. In H. Boege \& H.-J.P. Walter (Hrsg.).(2008), 34-61.

Duncker, K. (1935). Zur Psychologie des Produktiven Denkens. Berlin: Julius Springer.

Ehrenfels, v. Chr. (1890): Über „Gestaltqualitäten“. In F. Weinhandl (Hrsg.) (1974), Gestalthaftes Sehen. Ehrenfels-Festschrift (S.11-43). Darmstadt: Wissenschaftliche Buchgesellschaft.

Ehrenfels, v. Chr. (1937). Über Gestaltqualitäten. In Chr. v. Ehrenfels (1988). Psychologie, Ethik, Erkenntnistheorie. Philosophische Schriften, Bd. 3 (S. 168-170). Herausgegeben von Reinhard Fabian. München/ Wien: Philosophia Verlag. Auch in F. Weinhandl (Hrsg.). (1974³), S. 61-63.

Ehrenfels, v. Chr. (1922). Weiterführende Bemerkungen (in Das Primzahlengesetz, 95-112). InF. Weinhandl (Hrsg.).(1974), S. 47-60.

Ehrenfels, v. Chr. (1916). Höhe und Reinheit der Gestalt(in Kosmogonie, 93-96). In F. Weinhandl (Hrsg.) (1974), S. 44-46.

Goerlich, St. (2000). „Auf die 'Sachen selbst' zurückgehen“ - Berührungspunkte zwischen philosophischer Phänomenologie und gestalttheoretischer Psychotherapie. Gestalt Theory 22, 1, 47-46.

Gollwitzer, H. (1963). Die Existenz Gottes im Bekenntnis des Glaubens. München: Chr. Kaiser Verlag.

Hartmann, N. (19655', Erstaufl. 1921). Grundzüge einer Metaphysik der Erkenntnis. Berlin: de Gruyter. (im Text: ME)

Hartmann, N. (1933, 1949, 1962). Das Problem des geistigen Seins. Berlin: de Gruyter. (im Text: GS)

Hartmann, N. (1939, 19643). Der Aufbau der realen Welt. Berlin: de Gruyter. (im Text: RW)

Hartmann, N. (19493). Ethik. Berlin: de Gruyter.

Hartmann, N. (1954³). Einführung in die Philosophie. Überarbeitete, vom Verf. genehmigte Nachschr. der Vorlesung im SS 1949 in Göttingen. Osnabrück: Luise Hanckel Verlag.

Hartmann, N. (1955, 1957, 1958). Kleinere Schriften. Bde I-III. Berlin: de Gruyter.

Hartmann, N. (1966). Teleologisches Denken. Berlin: de Gruyter.

Hartmann, Nicolai \& Heimsoeth, Heinz im Briefwechsel. (1978). (hrsg. v. Frida Hartmann und Renate Heimsoeth). Bonn: Bouvier Verlag Herbert Grundmann.

Hartmann, N. (1949). Die Erkenntnis im Lichte der Ontologie. In Kleinere Schriften, Bd. I,(1955), 122-180. Berlin: de Gruyter.

Hartung, G. \& Wunsch, M. (Hrsg).(2014). Nicolai Hartmann, Studien zur Neuen Ontologie und Anthropologie. Berlin/Boston: de Gruyter.

Husserl, E. (1913² Nachdruck der 2. Aufl.: 19685).Logische Untersuchungen, Band II: Untersuchungen zur Phänomenologie und Theorie der Erkenntnis. Tübingen: Max Niemeyer.

Klages, L. (1923). Ausdrucksbewegung und Gestaltungskraft. Leipzig. Ambrosius Bart.

Klages, L. (1936). Handschrift und Charakter. Leipzig. Ambrosius Barth.

Koffka, K. (1915). Zur Grundlegung der Wahrnehmungspsychologie. Eine Auseinandersetzung mit V. Benussi. Ztschr. f. Psychologie und Physiologie der Sinnesorgane 73, 11-90.

Köhler, W. (1924). Gestaltprobleme und Anfänge einer Gestalttheorie. In Jahresberichte über die gesamte Physiologie und experimentelle Physiologie für das Jahr 1922, 3, 512-539. Reprint in Gestalt Theory 5, 3, (1983), 179-205.

Köhler, W. (1968). Werte und Tatsachen. Berlin: Springer.

Meinong, A. (1904). Über Gegenstandstheorie.Verfügbar unter http://www.gleichsatz.de/b-u-t/gene/meinong/ standtheorie l.html [Abruf: 12.07. 2016].

Meinong, A. (1918). Zum Erweise des allgemeinen Kausalgesetzes. Wien: Kais. Akad. der Wissenschaften. 


\section{GESTALT THEORY, Vol. 40, No.3}

Meinong, A. (1965). Philosophenbriefe. Hrsg. und mit Anmerkungen versehen v. Rudolf Kindinger. Graz: Akad.

Druck- und Verlagsanstalt.

Metzger, W. (1962). Schöpferische Freiheit. Frankfurt: Kramer.

Metzger, W. (1963). Psychologie. Darmstadt: Steinkopff.

Metzger, W. (1966a): Der Ort der Wahrnehmungslehre im Aufbau der Psychologie. InHdb.der Psychologie, Bd. 1, 1. Halbbd.:Wahrnehmung und Bewußtsein, 3-20. Göttingen: Hogrefe.

Metzger, W. (1966b): Figural-Wahrnehmung. In: siehe 1966a, 693-744.

Rausch, E. (1966a). Probleme der Metrik (Geometrisch-optische Täuschungen). In Hdb. d. Psychologie, Bd. 1, 1. Halbbd.:Wahrnehmung und Bewußtsein, 776-865. Göttingen: Hogrefe.

Rausch, E. (1966b): Das Eigenschaftsproblem in der Gestalttheorie der Wahrnehmung. In: siehe 1966a, 866953. Göttingen: Hogrefe.

Scheler, M. (1929). Philosophische Weltanschauung. Bonn: Verl. v. Friedrich Cohen.

Stegmüller, W. (1975). Hauptströmungen der Gegenwarts-Philosophie. Stuttgart: Kröner.

Tholey, P. \& Utecht, K. (1987). Schöpferisch träumen. Niedernhausen/Ts.: Falken-Verlag.

Tholey, P. (1992). Der ökologische Ansatz der Umweltwahrnehmung - ein Beitrag zur semantischen Umweltverschmutzung. Gestalt Theory 14, 2, 115-142 (Teil I) und 3, 197-218, (Teil II).

Toccafondi, F. (2003). Aufnahme, Lesarten und Deutungen der Gestaltpsychologie. Gestalt Theory 25, 3, 139-157.

Walter, H.-J.P. (1988). Sind Gestalttheorie und Theorie der Autopoiese miteinander vereinbar? Eine polemische Erörterung am Beispiel des STADLER/KRUSEschen Kompilierversuchs. Gestalt Theory 10, 1, 57-70; auch enthalten in Walter (1996).

Walter, H.-J.P. $\left(1985^{2}, 1994^{3}, 2018^{4}\right)$. Gestalttheorie und Psychotherapie. Opladen: Westdeutscher Verlag; neu: 4. Aufl. Remscheid: Rediroma Verlag.

Walter, H.-J.P. (1996). Angewandte Gestalttheorie in Psychotherapie und Psychohygiene. Opladen: Westdeutscher Verlag. Verfügbar auch unter http://www.gestalttheorie-dagp.de/index.php/literatur/publikationen-des-ehrenvorsitzenden-der-gta

Walter, H.-J.P. (2011/12). Das „Ich“ als Widersacher der Dummheit. Int. Ztschr. für Sozialpsychologie und Gruppendynamik 36, 2, 6-30 (Teil I) und 37, 1, 20-42 (Teil II). Verfügbar auch unter http://www.gestalttheorie-dagp.de/images/pdf/dumm.pdf

Walter, H.-J.P. (2012). [Das] Abenteuer [im] Kopf. Viertklässler treiben Erkenntnistheorie. Unveröff. Ms.

Walter, H.-J.P. (2016). Karl Duncker „meets“ Nicolai Hartmann. Gestalt Theory 38, 1, 5-15.

Weinhandl, F. (Hrsg.). (1960, 1974³). Gestalthaftes Sehen. Zum hundertjährigen Geburtstag von Christian von Ehrenfels. Darmstadt: Wissenschaftl. Buchgesellsch.

Wendelborn, S. (2003). Der Gestaltpsychologe Karl Duncker. Frankfurt: Lang.

Wertheimer, M. $\left(1964^{2}\right)$. Produktives Denken. Frankfurt a.M.: Waldemar Kramer.

Wirth, Ingeborg (1965): Realismus und Apriorismus in Nicolai Hartmanns Erkenntnistheorie. Berlin: de Gruyter.

Wunsch, Matthias: persönliche Mitteilung per E-Mail v. 05.06. 2017 nach Zusendung der Fassung dieser Arbeit vom Vortag.

Hans-Jürgen P. Walter, geb. 1944, Dr. phil., Dipl.-Psychologe, Psychotherapeut, Begründer der Gestalttheoretischen Psychotherapie; 1978 Mitbegründer der internationalen Gesellschaft für Gestalttheorie und ihre Anwendungen GTA, langjähriger 1. Vorsitzender, seit 1999 Ehrenvorsitzender; Mitgründer der internationalen multidisziplinären Zeitschrift Gestalt Theory. Lehrtätigkeit an der Universität Wien sowie Lehrtherapeut in zahlreichen psychotherapeutischen Vereinigungen, besonders der Deutschen (DAGP) und der Österreichischen Arbeitsgemeinschaft für Gestalttheoretische Psychotherapie (ÖAGP). Autor zweier Grundlagen-Werke zur Gestalttheoretischen Psychotherapie („Gestalttheorie und Psychotherapie“ und „Angewandte Gestalttheorie in Psychotherapie und Psychohygiene“) sowie zahlreicher Aufsätze.

Adresse: Battenbergerstrasse 1, 35216 Biedenkopf/Lahn, Deutschland.

E-mail: rottrecht@t-online.de 